Voix et Images

voixetimages

\title{
Sept incipit des années 1930
}

\section{Lori Saint-Martin}

Volume 39, numéro 2 (116), hiver 2014

Voix de femmes des années 1930

URI : https://id.erudit.org/iderudit/1025186ar

DOI : https://doi.org/10.7202/1025186ar

Aller au sommaire du numéro

Éditeur(s)

Université du Québec à Montréal

ISSN

0318-9201 (imprimé)

1705-933X (numérique)

Découvrir la revue

Citer ce document

Saint-Martin, L. (2014). Sept incipit des années 1930. Voix et Images, 39(2),

17-23. https://doi.org/10.7202/1025186ar d'utilisation que vous pouvez consulter en ligne.

https://apropos.erudit.org/fr/usagers/politique-dutilisation/ 


\title{
D O C U M E N T
}

\author{
Sept incipit des années 1930 \\ $++$ \\ préparé par \\ LORI SAINT-MARTIN \\ Université du Québec à Montréal
}

Ils ne circulent plus, ces romans, et plus personne ne connaît le son de ces voix. Comment étaient-elles, que disaient-elles? Voici sept débuts ${ }^{1}$, choisis en fonction d'un critère bien subjectif, le plaisir de lecture qu'ils procurent, qui illustrent le travail de quelques femmes étudiées dans le présent numéro (Éva Senécal, Jovette-Alice Bernier, Michelle Le Normand, Laure Berthiaume-Denault) et de certaines de leurs contemporaines. Parfois accomplis, parfois maladroits, ces romans présentent, dès leurs premières lignes, au moins une vie de jeune femme en devenir, une jeune femme ardente, ouverte, curieuse devant la vie et les êtres, et souvent sur le point de vivre une aventure qui lui révélera sa vraie nature ou celle du monde qui l'entoure. La promenade dans une nature qui reflète et révèle les sentiments intimes, l'amitié entre femmes, la culture (peinture, théâtre, voyages...) sont des motifs récurrents. On notera aussi l'ambiance joyeuse des milieux bourgeois - thêatres, hôtels, lieux de villégiature - , les évocations sensuelles, la présence de figures «exotiques" (l'Américain, le «Sauvage»), la recherche constante de contrastes : entre des amies, entre un mari ou un fiancé terne et un amoureux aux charmes multiples, entre une jeune femme et une épouse ou une grand-mère résignée. Malgré les yeux qui surveillent, les héroïnes romanesques affichent leur liberté de pensée et de mouvement. Aujourd'hui oubliés, ces romans méritaient pourtant mieux.

\section{ÉVA SENÉCAL, DANS LES OMBRES ${ }^{2}$}

D'un pas lent, la jeune femme quitta sa retraite et revint vers la maison, à demi cachée dans les ombres de la nuit venue.

1 À part deux corrections évidentes («eût», "fût»), nous avons choisi de reproduire les textes tels quels.

2 Montréal, Éditions Albert Lévesque/Librairie d'Action canadienne-française, coll. «Les romans de la jeune génération », 1931, 150 p. 
Toujours le même tableau. Les deux mêmes êtres se trouvaient toujours aux mêmes places. Immobiles dans de larges fauteuils de cuir brun, éclairés à peine par un reflet de la lampe que voilait l'abat-jour, ils ressemblaient à deux sphinx. Mais que lui importait ce soir! Elle en avait déjà trop du spectacle des êtres et des choses. Elle ressentait le besoin de voir en elle, de ne voir qu'elle.

Entrée sans bruit, elle gravissait déjà l'escalier pour se rendre à sa chambre quand le vieillard, de son fauteuil, l'arrêta:

- Il vient d'arriver une lettre pour toi, Camille, dit-il.

Du geste, il lui désignait une enveloppe blanche posée sur le bord de la fenêtre. Elle la prit, regarda le timbre.

Rouyn.

Rien de nouveau. Elle en recevait ainsi chaque semaine et toutes disaient la même chose. Comme il savait mal les écrire, ces lettres qu'elle eût voulues autrement, plus simples, avec moins de phrases toutes faites où elle cherchait en vain son âme qui ne devait pourtant pas être comme les autres.

Que n'essayait-il, avec des mots enthousiastes, évocateurs, de l'attacher un peu aux mille détails de sa vie, à ces paysages lointains qui seraient un jour «leurs » paysages! Que ne savait-il la charmer avec des notes accordées aux résonnances du cœur, palpitantes de rêves épars, des riens de chaque jour magnifiés par sa tendresse.

Mais non. Il ne savait pas. Il vivait loin d'elle et s'en éloignait un peu plus par chacune de ses lettres.

Elle se reprit tout à coup, sentant qu'on l'observait. Et l'on ferait encore des reproches, on parlerait de son indifférence. C'était aussi dans le programme de ses journées. Elle s'y habituerait peut-être.

- Quel est cet étranger avec qui je t'ai vue revenir du lac? demanda tout à coup sa grand'mère.

JOVETTE-ALICE BERNIER, LA CHAIR DÉCEVANTE ${ }^{3}$

Lombreval, 25 juillet $19 . .$.

À monsieur Jean Vader,

Montréal.

Ce que je fais ici? je regarde, tout le jour, si tu viens; je m'inquiète quand le soir arrive tout seul. Pour te sourire avant lui, je me lève avant le soleil; je passe mes journées sur la grève; je bois tout l'été qui passe, et je m'ennuie.

Les nuits sont trop belles, ce qui t'explique pourquoi je dors sur les galets dans les chaudes matinées. Je m'ennuie, et j'ai ennuyé la lune à force de la regarder s'ar-

3 Montréal, Éditions Albert Lévesque/Librairie d'Action canadienne-française, coll. «Les romans de la jeune génération », 1931, 137 p. 
rondir sur la mer; j'ai importuné les oiseaux... ils ne comprennent pas qui est cette folle qui sommeille à midi et qui s'oublie sur son balcon, la nuit.

Viens. Tu ne me reconnaîtras plus bientôt: je suis basanée jusqu'aux chevilles, et il paraît que je fais pitié dans du blanc. Viens. Si je désapprenais à rire, où trouveraistu les traits de ta Didi, ensuite? Viens, pour que j'aime mieux tout ce que j'aime.

Didi.

L'heure du bain. Maillots bigarrés, unicolores, têtes diverses, rires divers. Toute cette jeunesse qui s'ébroue, et la belle marée qui s'effrange. Détente voluptueuse des corps. Des enfants se mêlent, timides.

Là-bas, ce globe de feu qui va tomber dans l'eau et qui ne s'éteindra jamais; qui flamboiera demain sur la montagne de l'autre côté... Des barques. C'est cela la vie reposante?

... Non, ce n'est pas cela la vie reposante. Ce que c'est, au juste, je ne sais pas; il y a des gens qui la portent en eux, pour d'autres, et qui ne s'en soucient pas; il y a des gens qui fuient avec toute la tendresse qu'ils nous doivent.

Il y a une épaule où j'ai égaré la joie que j'avais autrefois, et cette épaule a gardé la consolation que j'y devais prendre en retour. Il y a des bonheurs qui sont faits pour nous et que d'autres emportent sans le savoir. Tout cela il faut le rebâtir ailleurs.

Le jour où j'aurais le courage d'avouer, j'aurais le droit de ma franchise; ah! la surprise de rencontrer son regard qui ne me mépriserait pas, si je parlais...

Jean viendra demain. Sa lettre est sereine, confiante et calme au point de donner le vertige ; c'est comme une grande plaine sans accidents où le regard voit trop de pareil.

Je voudrais qu'il me devine, qu'il m’accuse, qu'il me fasse mal. Cette quiétude envers moi me désempare. Il m'aime pour ce que je devrais être et que je ne suis pas ; pour un passé qu'il me fait selon son rêve d'homme fanatisé.

Je lui pardonnerais qu'il fût un traître, un assassin...

Mais Jean n'a rien à se faire pardonner que des peccadilles qui n'en sont même pas à mes yeux.

Jean a l'âme que j'avais à seize ans.

Ah! l'orgueil des yeux qui n'ont pas péché. L'exigence du cœur qui ne se reproche rien.

J'ai dit mille choses futiles pour éviter le sujet nécessaire; j'ai été légère pour ne pas paraître soucieuse; pour ne pas paraître songeuse, j'ai fait de si distraites réflexions pour me ressaisir... que j'ai honte de l'âme montrée à l'envers.

\section{MARIE-ROSE TURCOT, UN DE JASPER ${ }^{4}$}

Le Jasper-Lodge regorge de touristes. Hôtel spacieux qui, dans un décor rustique, offre l’hospitalité la plus cordiale et un confort élégant.

$$
+++
$$

4 Montréal, Éditions Albert Lévesque/Librairie d'Action canadienne-française, 1933, 168 p. 
Ce citadin, en complet de Montagnard, c'est Richard Tellier, le peintre, qui doit couvrir de fresques le pavillon destiné à son excellence le Gouverneur Général du Canada.

Richard Tellier a fait un séjour de deux années en Europe à l'école des grands maîtres.

- Un peu poseur, remarque une étrangère à sa compagne, qui vient de lui désigner le peintre.

- Bon camarade, cependant, et charmant, souligne l'autre.

Cette autre, c'est Esther Joron, la femme du guide suisse. Elle agit comme cicerone et joue le rôle d'hôtesse auprès des arrivants. Elle les initie aux beautés de Jasper et trace à leur intention l'itinéraire qui devra rendre leur séjour intéressant: des ascensions vers les sommets, des excursions de chasse ou de pêche, dont elle laisse les responsabilités à son mari.

À l'extrémité du couloir, ouvrant sur la rotonde de l'hôtel, deux jeunes femmes s'avancent à la rencontre de Richard Tellier. L'aînée, Charlotte, est toujours au pas de course. (Est-ce bien l'aînée des deux, cette figurine de Delft, poupine et rose, et sans âge apparent, mais non sans grâce?) Elle sait faire valoir l'agilité de son pied de nymphe. Saisissant le bras de son frère qui s'apprête à sortir, encombré d'une boîte de couleurs, d'un chevalet replié et d'un album de croquis:

- Richard, lui dit-elle, il faudra être exact pour le dîner, ce soir. Le gérant tient à te faire rencontrer un Américain, collectionneur d'œuvres d'art. Tu auras tout à y gagner, en l'édifiant par ton savoir-faire et par ta sagesse.

\section{LUCIE CLÉMENT, EN MARGE DE LA VIE ${ }^{5}$}

Il neige. Une langueur descend du ciel avec les flocons en forme d'étoiles et se pose, en effleurement, sur les fourrures des femmes qui se pressent à la sortie du Little Theatre. Ce thêâtre doit son cachet à l'atmosphère religieuse dont ses murs, verts et or, semblent imprégnés. Malgré le rideau de soie, qui se nuance de mille tons sous les feux de la rampe, cette salle reste temple protestant; l'auditoire y applaudit avec discrétion - elle sent l'incongruité du délire en un tel lieu - et aux entr'actes, les silences se prolongent.

La porte en ogive déverse le flot des spectateurs qui aspirent l'air avec délectation. Une à une, les automobiles se mettent en marche et le bruit des moteurs détonne dans les rues de la capitale du Dominion, qui bientôt retombe dans son calme habituel.

Sur les coussins de velours de l'une des dernières voitures s'installent, volubiles, deux jeunes filles.

$$
+++
$$

5 Montréal, Éditions Albert Lévesque, coll. «Romans canadiens», 1934, 192 p. 
Nicole Berteuil et Joan Webb obéirent, sans doute, à la loi des contrastes lorsqu'elles se lièrent d'une étroite amitié.

Différentes, certes, elle le sont au physique et combien plus au moral. Joan Webb est grande, très blonde, les yeux largement ouverts sur la vie qu'elle ne redoute pas, confiante en ses forces de sportive intellectuelle. Il y a chez Nicole Berteuil, au visage étroit, une ardeur de vivre aussi grande que chez son amie; une appréhension de l'avenir se loge cependant sous son front bombé. Impatiente, passionnée, elle s'irrite lorsque les circonstances s'érigent en obstacle sur sa route.

Cultivées, elles le sont toutes les deux; mais là encore, elles diffèrent.

\section{MICHELLE LE NORMAND, LA PLUS BELLE CHOSE DU MONDE ${ }^{6}$}

Ce soir-là, elles veillaient chez Monique. Depuis quelque temps, sous prétexte d'examens à préparer, une fois par semaine, elles se réunissaient chez l'une ou chez l'autre. Dans deux mois, elles auraient terminé leurs études. Toutes les quatre s'en réjouissaient. Elles n'étaient pourtant pas paresseuses, elles avaient l'esprit ouvert, avide, mais qu'elles étaient lasses des règlements à suivre, lasses d'être petites filles. Les romans leur donnaient un avant-goût de la vie. Impatientes, curieuses, ardentes, elles aspiraient à sortir au plus vite de leur chrysalide.

Lucette était arrivée la première. Elle ressemblait à une montre qui avance; elle ne pouvait se corriger d'arriver trop tôt. Nicole, au contraire, ne parut que dix minutes après l'heure fixée. Et Claire ne se montrant pas, Monique murmurait: chez elle.

- Si elle ne vient pas, la malheureuse, et sans me prévenir, je n'irai plus jamais

Un peu plus et elle aurait ajouté, comme dans un ancien temps tout proche, cette classique menace enfantine:

- Je ne lui parlerai plus.

Mais on sonna et justement c'était Claire. Elles dirent toutes ensemble des paroles de bienvenue. Puis, lorsqu'elles furent bien installées, autour d'un gros pouf sur lequel elles allongèrent commodément leurs jambes, Monique, sans raison, demanda :

- Quelle est, mes amies, la plus belle chose du monde?

Et elle répéta plusieurs fois cette phrase et les pressa d'y répondre.
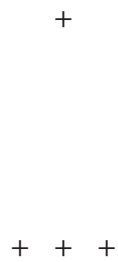

6 Montréal, Éditions du Devoir, 1937, 249 p. 
Je m'appelle Linette Geoffrion. Je n'avais que trois ans lorsque ma mère mourut me laissant à mon père, et sous les soins d'une tante. J'étais alors assez vieille pour comprendre, et pour connaître toutes les tendresses qu'une mère peut avoir pour son enfant; je réalisai donc aussitôt qu'une nouvelle vie s'ouvrait pour moi.

Mon père s'absentait assez souvent et pour de longs voyages même quelquefois. Il me laissait en pension ou sous les soins de ma tante qui, avant tout n'entendant que son confort, me laissait me débrouiller seule. Parfois, c'était un peu dur pour moi. Ma petite intelligence commençait à comprendre dès lors que ce que j'avais de plus cher au monde m'avait été enlevé.

Pourtant, à cet âge, on ne doit pas mériter une si grande épreuve, mais c'était la volonté de Dieu et personne ne peut rien contre Lui.

Chaque été, une dame assez âgée venait passer quelques mois dans notre village; et je vous ai déjà dit que mon père pendant ses longues absences me mettait en pension à l'hôtel pour plus de sécurité; alors c'est là que je fis la connaissance de cette dame (Madame Jacques), qui s'intêressait beaucoup à ma petite personne.

Un jour que mon père vint me chercher à l'hôtel, elle demanda à le voir; mon père se fit un plaisir de répondre à son invitation. Puis, de jour en jour, elle s'intéressait à moi davantage, ce qui plaisait beaucoup à papa.

Un jour qu'ils étaient en conversation, mon père lui fit entendre qu'il aimerait bien à me voir placée dans une bonne institution où je pourrais recevoir une bonne instruction et surtout une bonne éducation, afin que je puisse marcher seule dans la vie, étant orpheline.

Alors, Madame Jacques, toute contente, s'empressa de lui dire que ce serait pour elle un plaisir de m'accompagner chemin faisant, et de s'occuper de me placer dans un couvent de Québec, puis elle dit à mon père qu'elle ferait tout pour me rendre heureuse.

\section{LAURE BERTHIAUME-DENAULT, MON SAUVAGE ${ }^{8}$}

La première vanne ouverte, le Wilfrid-Laurier glissa lentement dans le canal Rideau vers la rivière Ottawa. Un matelot sauta agilement sur le talus de l'écluse pour saisir les câbles, puis le cargo descendit doucement avec l'eau. La sirène, d'un coup bref, annonça: «Lâchez les câbles!» Le capitaine, les mains derrière le dos, arpentant la passerelle, suivait attentivement la manœuvre. La deuxième vanne s'ouvrit.

Liliane, en simple tailleur et béret bleu marine, se tenait à l'arrière du pont, à côté de son hôtesse, $\mathrm{M}^{\mathrm{me}}$ Ranger, l'épouse du capitaine.

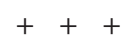

7 Québec, Éditions de l'Action canadienne-française, 1938, 76 p.

8 Montréal, Éditions Bernard Valiquette, 1938, 216 p. 
- La fraîcheur de l'eau vient jusqu'à nous, dit Liliane. Aspirant longuement, elle continua: J'ai le pressentiment que je vais avoir beaucoup de plaisir durant cette excursion. N'est-ce pas que c'est amusant?

- Ah! oui, pour vous. Moi, j'y suis tellement habituée... Depuis quinze ans, tous les étés, je voyage ainsi avec mon mari, d'Ottawa à Montréal. J'aime beaucoup ce genre de vie, mais ce que je trouve long, c'est le passage des écluses. On prendra bien une heure à passer celle-ci. Pour vous, c'est amusant; le nouveau a toujours de l'attrait.

- J'aime l'aventure. Fillette, rien ne me rendait plus heureuse que d'entendre ma mère m'annoncer que nous allions visiter ses parents en France. Je me rappelle trois beaux voyages, mais le dernier n'a pas été aussi agréable pour moi. J'avais pourtant dix-sept ans et ma mère, au lieu de me mener au bal à bord, après dîner, me renfermait dans ma cabine. "Tu n'es pas d'âge à te mêler aux jeunes gens. Sur un paquebot, on ne sait jamais au juste qui l'on coudoie, à moins que ce ne soient de vieilles connaissances. » Et à Paris donc! Nous n'y allions que pour nos emplettes, ou, le soir, pour le thêâtre. Mais, moi, j'aurais tant aimé voyager seule; visiter les ateliers d'artistes, les cabarets montmartrois, errer dans les petites rues mystérieuses! Je rêvais de Louise et Julien que je venais de voir à l'Opéra. Ces idées effrayaient ma mère. Aussi, cette année, quand elle me proposa de l'accompagner, j'ai préféré rester à Ottawa. Maman doit revenir en septembre.

- Je ne dédaignerais pas un voyage en Europe, répondit $\mathrm{M}^{\mathrm{me}}$ Ranger, et à Paris surtout; mais quand on a plusieurs petites bouches à nourrir, on ne peut guère se payer ce luxe. Voilà pourquoi je me contente de notre belle grande rivière.

- Ah! qu'il y fait bon! s'écria Liliane, en regardant le rideau de verdure bordant la colline du Parlement. Regardez le Chemin des Amoureux. J'ai souvent demandé à mon fiancé de m'y conduire, mais il prétend qu'une jeune fille bien élevée ne fréquente pas ces allées ombreuses.

- Vous êtes fiancée?

- Oui, à un ami de la famille, riche et distingué.

- Alors, c'est le parfait fiancé!

- Oh, oui ! le fiancé idéal, comme dit ma mère.

- N'est-il pas votre idéal, à vous aussi?

- Peut-être... Il a vingt ans de plus que moi, mais il est accompli! Et maman m'assure qu'il sera un bon mari.

- Vous n'avez jamais eu d'autre ami?

- Quand j'étais fillette, je recevais d'un correspondant des billets doux auxquels je répondais. Il s'appelait Armand et apprenait le violon.

Faisant quelques pas sur le pont, Liliane aperçut un grand jeune homme au teint bronzé qui la regardait.

- Qui est-il ? murmura-t-elle à $\mathrm{M}^{\mathrm{me}}$ Ranger.

- C'est un étudiant de l'Université de Montréal, un métis.

- Tiens, un sauvage !... Il étudie à Montréal?

- Dans un an il sera avocat.

- Avocat! Pour un sauvage, ça n'est pas commun. 